\title{
Autenticidade científica de um teste de agilidade para o voleibol sentado
}

\section{Scientific authenticity of an agility test for the sitting volleyball}

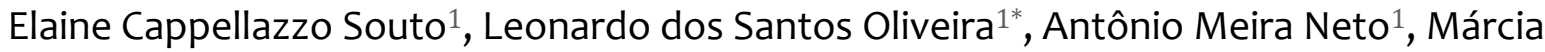 \\ Greguol $^{2}$
}

\begin{abstract}
RESUMO
Este estudo investigou a confiabilidade teste e reteste, confiabilidade interavaliador e validade de conteúdo de um Teste de Agilidade para o Voleibol Sentado (TAVS). Oito atletas com deficiência motora (44 \pm 12 anos) foram avaliados por 4 examinadores em 2 diferentes ocasiões, percorrendo um trajeto de 6 metros em forma de "T", na posição sentada e em máxima velocidade. A confiabilidade foi analisada por meio do coeficiente de correlação intraclasse $(C C I)$, do método de Bland-Altman e por testes de comparação nãoparamétricos, com $95 \%$ de confiança. Para a validação de conteúdo, cinco especialistas analisaram a clareza da descrição e aplicabilidade do teste para a modalidade. Os tempos do teste foram similares entre as duas ocasiões $(p=0.19)$. A confiabilidade do TAVS foi muito boa, com $C C I>0.90$ e baixo erro. O TAVS apresentou alta confiabilidade e a análise da clareza da descrição e aplicabilidade do teste pelos especialistas demonstrou validade de conteúdo, indicando que a proposta é capaz de mensurar a agilidade mediante um protocolo de campo, de baixo custo e de fácil aplicação nos contextos indoor para o voleibol sentado. Palavras-chave: Desempenho Psicomotor, Desempenho Atlético, Avaliação da Deficiência.
\end{abstract}

\begin{abstract}
This study investigated the test-retest reliability, inter-rater reliability and content validity of an Agility Test for the Sitting Volleyball (ATSV). Eight athletes with physical disabilities $(44 \pm 12$ years) were evaluate by 4 examiners from 2 separate trials, covering a 6 -meter T-shaped course in the sitting position and at maximal speed. Reliability was analyzed using the intraclass correlation coefficient (ICC), BlandAltman method and nonparametric tests for comparisons, with 95\% confidence. For content validity, five specialists analyzed description clarity and test applicability. The test times were similar in both occasions $(p=0.19)$. Reliability of ATSV was very good, with $I C C>0.90$ and low bias. The ATSV showed high reliability and expert analysis of description clarity and test applicability demonstrated content validity, suggesting that the proposal is capable of measuring the agility through a low-cost and easy-to-apply field protocol in indoors contexts of sitting volleyball.
\end{abstract}

Keywords: Psychomotor Performance, Athletic Performance, Disability Evaluation.

\footnotetext{
Artigo recebido a 16.07.2014; Aceite a 09.03.2015

${ }^{1}$ Universidade Federal da Paraíba - UFPB, João Pessoa, Brasil

${ }^{2}$ Universidade Estadual de Londrina - UEL, Brasil

* Autor correspondente: Leonardo dos Santos Oliveira. Laboratório de Cineantropometria - Centro de Ciências da Saúde - Universidade Federal da Paraíba. Cidade Universitária - João Pessoa/PB - Brasil - CEP 58051900 E-mail: leonsoliveira@gmail.com
} 


\section{INTRODUÇÃO}

Desde sua inserção nos Jogos Paralímpicos, em 1980 na Holanda, o voleibol sentado tem se desenvolvido em todos os continentes, trazendo uma demanda substancial para uma análise do desempenho atlético confiável, precisa, adequada e viável para os técnicos (Medeiros, Ribeiro, \& de Oliveira, 2012). No desporto paralímpico, a quantidade de interferência da deficiência motora sobre a função específica em cada modalidade define a classificação funcional do atleta (Tweedy \& Vanlandewijck, 2011). Todavia, poucos são os instrumentos de avaliação que levam em consideração a especificidade da deficiência (Brasile, 1986, 1990; Gorgatti \& Böhme, 2003; Yilla \& Sherrill, 1998).

Sabe-se que a aplicação de testes específicos para avaliar componentes da aptidão física relacionada à performance é uma prática frequente para identificar o real estado de treinamento do atleta, elaborar treinos adequados e identificar talentos desportivos. Além de outros componentes da aptidão física relacionados à performance (força, potência, flexibilidade, velocidade), a agilidade é fundamental para o treinamento do voleibol sentado (Vute \& Urnaut, 2009), sendo um componente essencial na maior parte dos desportos de campo e equipa (Sheppard \& Young, 2006). Embora pesquisadores na área do desporto não apresentem um consenso sobre sua definição, a agilidade é, classicamente, definida como a capacidade de mudar de direção rapidamente (Sheppard \& Young, 2006). No voleibol sentado, esta ação é desempenhada pelos membros superiores dos atletas para deslocar seu corpo no solo em resposta ao movimento da bola e dos outros jogadores, em uma quadra com 10x6 metros, o que é uma particularidade da modalidade e merece a atenção especial dos treinadores.

$\mathrm{Na}$ literatura, trabalhos ainda escassos, em diferentes áreas, têm contribuído para o voleibol sentado. Na psicologia do desporto, o nível de identidade atlética e satisfação com a vida foi comparado entre atletas do voleibol sentado e convencional (Wiś niowska, Tasiemski, \& Bauerfeind, 2012), os fatores motivacionais foram analisados levando em consideração as especificidades dos atletas em relação à idade, aquisição da deficiência e tempo de treino (Protić \& Valkova, 2011). Na área do treino, foram investigados os efeitos do treinamento programado sobre as habilidades motoras de atletas (Hasanbegović, Ahmetović, \& Dautbasic, 2011), a relação entre a habilidade cognitiva e a motora específica (Soše, 2009), a avaliação da aptidão física e específica por meio de medidas antropométricas e testes físicos e motores (Molik, Kosmol, \& Skucas, 2008) e a análise do jogo em uma competição (Vute, 1999). Sabe-se que os testes de campo são uma maneira viável para obter uma indicação do padrão de desempenho. Entretanto, em relação à criação de um teste para avaliar as habilidades específicas no voleibol sentado, encontrou-se somente um estudo realizado em laboratório para verificar os efeitos de duas posições das mãos para o deslocamento (Singhal, Hwang, Davis, \& Kwon, 2013). Portanto, diante da necessidade de instrumentos de avaliação que levem em conta a especificidade do voleibol sentado, em condições de serem aplicadas em campo pelo treinador, a importância da agilidade para a execução da modalidade e o incipiente estudo na área, este estudo teve como objetivo investigar a confiabilidade teste e reteste, confiabilidade interavaliador e validade de conteúdo de um Teste de Agilidade para o Voleibol Sentado (TAVS).

\section{MÉTODO}

Trata-se de um estudo de validação, de corte transversal, com modelo de análise quantitativa (Nelson, Thomas, \& Silverman, 2012). O procedimento de verificação da autenticidade científica do TAVS foi composto pela determinação da confiabilidade teste e reteste, confiabilidade entre avaliadores, bem como da validade de conteúdo. Inicialmente, a aplicação do Teste de Agilidade para o Voleibol Sentado (TAVS) foi realizada em dois estágios. No primeiro estágio, testou-se a confiabilidade do TAVS (teste e reteste), com 48 horas de intervalo. No segundo estágio, verificou-se a confiabilidade entre avaliadores do TAVS, com aplicação do teste por quatro observadores distintos (A, B, C e D) (Figura 1). Os 
observadores eram técnicos de equipes de voleibol sentado, com curso de especialização na área, com no mínimo 1 ano de experiência com o voleibol sentado competitivo. Em adição, analisou-se o desempenho do deslocamento lateral (para a direita ou esquerda) no início do teste.

Posteriormente, realizou-se a validação de conteúdo com especialistas da área da Atividade Física Adaptada.

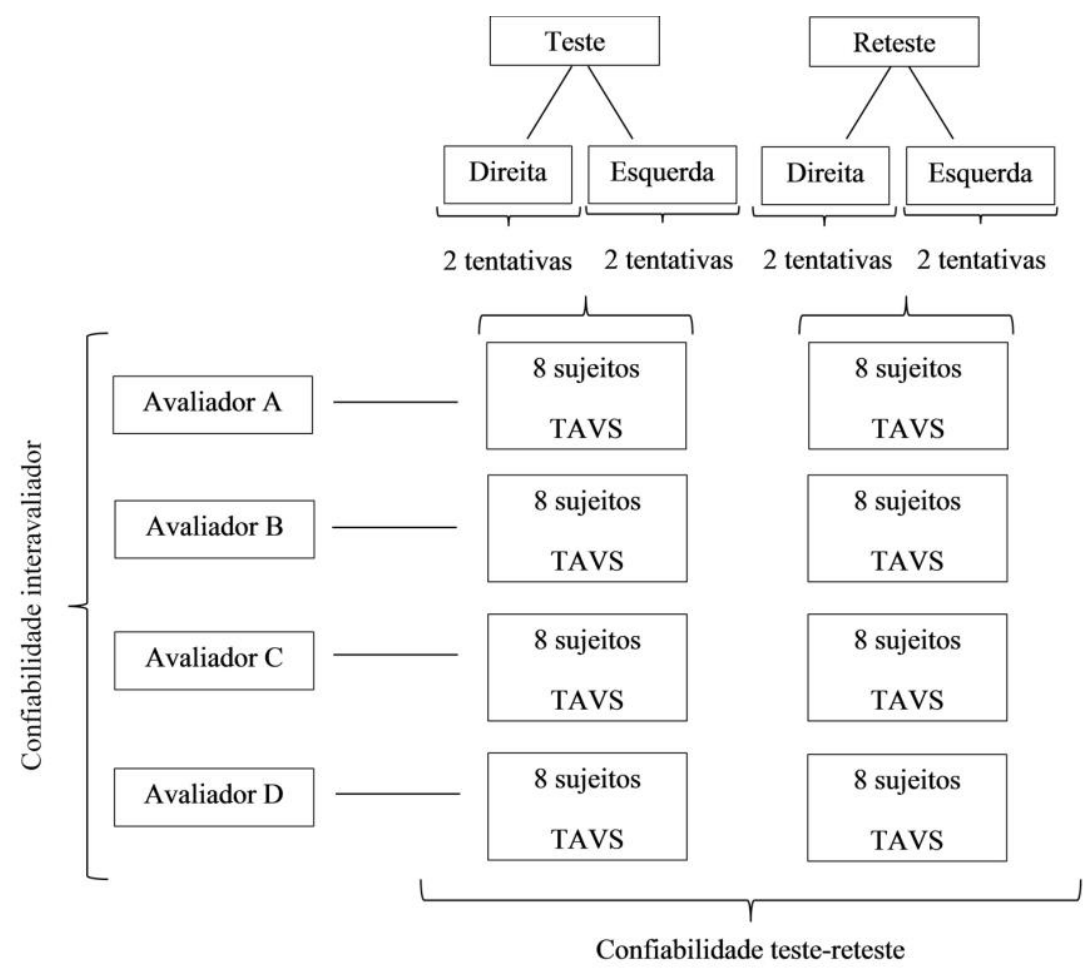

Figura 1. Desenho do estudo.

\section{Participantes}

Foram selecionados, de forma nãoprobabilística, oito participantes $(n=8)$, homens adultos (44 \pm 12 anos), com deficiência motora há, no mínimo, dois anos, verificando-se na amostra: amputação bilateral de membros inferiores $(n=2)$, amputação unilateral de membro inferior $(n=3)$, paraplegia $(n=2)$ e pé torto congênito $(n=1)$. Todos os voluntários eram atletas de voleibol sentado. Como critério de inclusão, os atletas deveriam ter deficiência motora nos membros inferiores, treinar voleibol sentado no mínimo duas vezes por semana, durante duas horas cada dia, há mais de um ano. Como critério de exclusão, estabeleceu-se a presença de deficiência motora nos membros superiores. Os sujeitos do estudo foram recrutados no "Núcleo de Esporte de Alto Rendimento para Pessoas com Deficiência", com sede na Universidade Federal da Paraíba (UFPB), que se voluntariaram para o estudo, assinando um termo de consentimento esclarecido, após serem informados dos objetivos e procedimentos desta pesquisa. Este estudo foi aprovado por um Comitê de Ética da UFPB.

\section{Procedimentos}

O procedimento inicial, para a criação do Teste de Agilidade para o Voleibol Sentado (TAVS), teve como base a análise das distâncias e velocidade de deslocamentos para a execução dos fundamentos do voleibol, durante o jogo pelos atletas. Em seguida, buscas na literatura foram realizadas para identificar testes de agilidade aplicados em diferentes tipos de desporto. Posterior a esta fase, decidiu-se adaptar o teste "T" proposto por Semenick (1990).

Os testes foram realizados em um ginásio esportivo adequado para a prática da modalidade, sendo aplicados nos mesmos horários do dia. Os tempos foram registrados, em milésimos de segundos, por cronômetros digitais (G-SWlb, 
Telva, China). O trajeto foi medido por trena de fibra de vidro (Y1730CM, Lukfin, EUA) com precisão em milímetros, delimitado com fita adesiva de $5 \mathrm{~cm}$. Além disso, para que o participante percorresse cada ponto do trajeto de forma completa, ultrapassando todo o quadril, delimitou-se, com uma fita adesiva de $2.5 \mathrm{~cm}$ um espaço de $30 \mathrm{~cm}^{2}$ em cada ponto do trajeto (Figura 2).
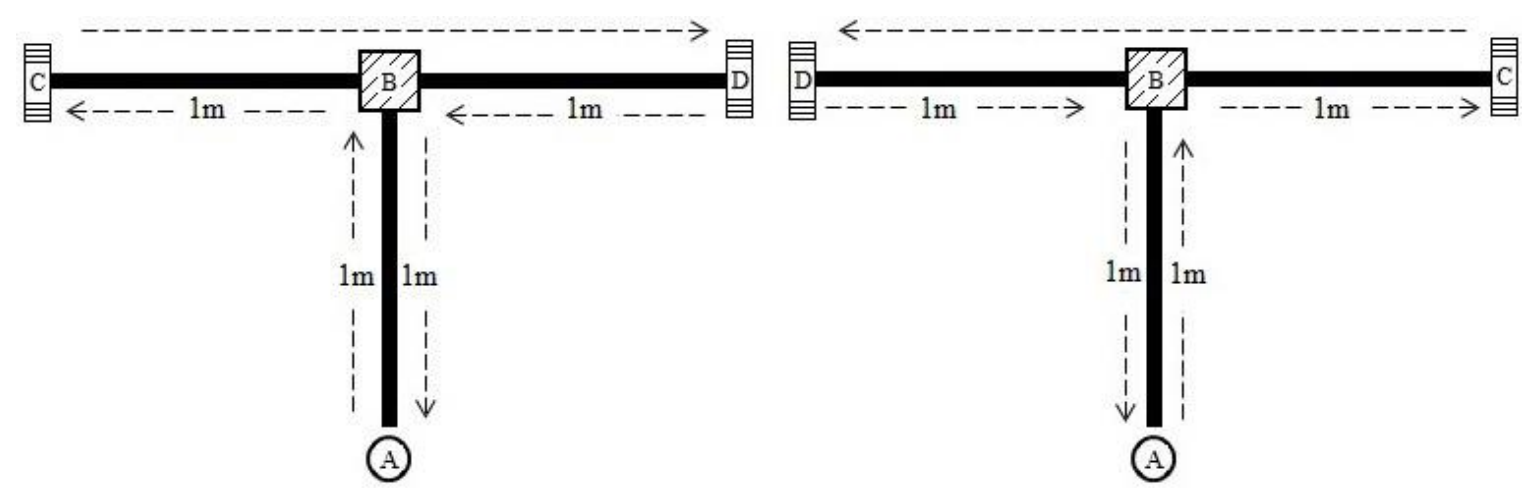

Figura 2. Layout do Teste de Agilidade para o Voleibol Sentado (TAVS), com início para o lado esquerdo e direito.

Cada atleta foi instruído uniformemente acerca do percurso do teste, inclusive informando-os a realizar o teste em esforço máximo. Padronizou-se o texto: "Este teste pretende medir a sua agilidade. Você deve realizar o teste na maior velocidade possível, de forma a completar o percurso passando pelo ponto ao centro, nas extremidades direita/esquerda, retornar ao centro e, por fim, retornar para a posição inicial." Antes de cada procedimento, houve 5 minutos de aquecimento moderado e, na sequência, cada participante realizou uma tentativa para familiarização do protocolo, a fim de reduzir o efeito do aprendizado motor. O teste foi iniciado após a comunicação: "Quando quiser, pode começar". Foi realizado encorajamento verbal, igualmente para todos os participantes, durante a realização do teste, repetidamente com as palavras: "Vai, vai, vai, mais rápido". Os participantes usaram roupas próprias para a prática do voleibol sentado durante todas as ocasiões.

\section{Protocolo do Teste de Agilidade para o Voleibol Sentado (TAVS)}

O TAVS consiste em percorrer um trajeto em formato de " $T$ ", em um percurso total de 6 metros, com mudanças de direção, na maior velocidade possível, estando o indivíduo na posição sentada. O sujeito inicia o exercício com todo o quadril atrás do ponto inicial $\mathrm{A}$, deslocase 1 metro em direção ao ponto B (deslocamento frontal). Após isso, mais 1 metro para esquerda até ultrapassar o quadril da marca indicada no ponto C (deslocamento lateral). Em seguida, o sujeito desloca-se 2 metros para a direita até o ponto D (deslocamento lateral) e, então 1 metro para a esquerda, retornando ao ponto $\mathrm{B}$ (deslocamento lateral), e retorna, 1 metro (deslocamento para trás), ao ponto de partida A. O cronómetro deve ser acionado somente quando o indivíduo ultrapassa o ponto A e finalizado no instante em que o indivíduo cruzou novamente este ponto, a fim de reduzir a influência do tempo de reação individual (Figura 2).

O lado que o sujeito iniciou o deslocamento lateral (Ponto B) foi randomizado, depois que cada indivíduo realizou duas tentativas para cada lado. O intervalo entre as tentativas foi de 3 minutos. Registrou-se o menor tempo tanto para as tentativas do lado esquerdo, quanto para o lado direito. Se, porventura, ocorresse algum erro de natureza técnica na execução das corridas, inviabilizando-se o prosseguimento do teste, o mesmo era cancelado e refeito após intervalo de 5 minutos.

\section{Validação de conteúdo}

Partindo do pressuposto que a validade de conteúdo é "a evidência da veracidade de um 
teste, baseada na decisão lógica dos procedimentos e de sua execução" (Morrow, Jackson, Disch, \& Mood, 2014), aplicou-se um formulário adaptado de Gorgatti e Böhme (2003) a cinco especialistas da área da Atividade Física Adaptada. Nesse formulário, foram analisadas as opiniões dos especialistas quanto à clareza da descrição do teste (se muito fácil/difícil de entender), sua aplicabilidade (em termos de espaço, materiais e adequação à modalidade de voleibol paralímpico, se muito viável ou inviável) e se o teste media o que se propunha (se sim ou não).

\section{Análise estatística}

Os escores de tempos foram reportados por mediana e amplitude interquartil (AIQ), uma vez que os dados mostraram-se não normais (Teste de Shapiro Wilk, $p<$ 0.05), embora a homogeneidade da variância não tivesse sido violada (Teste de Levene, $p>0.05$ ). A fim de avaliar diferenças na dominância do lado direito ou esquerdo para início do TAVS, bem como diferenças teste e reteste, aplicou-se o teste de Wilcoxon. A diferença entre os quatro avaliadores foi verificada pelo teste de KruskalWallis. O coeficiente de correlação intraclasse (método teste e reteste) e o coeficiente Alpha de Cronbrach foram usados para determinar a confiabilidade entre participantes do TAVS. Os limites de concordância e erro foram calculados a partir do método de Bland-Altman. Ainda, o coeficiente de concordância foi empregado para avaliar a precisão e acurácia dos pares de observações (Correction: A Note on the Concordance Correlation Coefficient, 2000). Para a validação de conteúdo, utilizou-se a frequência relativa (\%) das respostas dos especialistas. A significância estatística foi configurada para $p<0.05$. Os dados foram analisados pelo IBM ${ }^{\circledR}$ SPSS $^{\circledR} 20.0$ (IBM SPSS Inc., EUA) e pelo MedCalc 12.7.2.0 (MedCalc Software, Belgium).

\section{RESULTADOS}

Inicialmente, são apresentados os resultados relacionados à confiabilidade teste e reteste e à confiabilidade interavaliador e, por fim, sobre a validade de conteúdo. Os valores descritivos do tempo (em segundos) tanto para o teste quanto para o reteste, para cada avaliador, podem ser observados na Tabela 1. Quanto à identificação da dominância do lado direito ou esquerdo para início do teste, não foram observadas diferenças significativas, nem no teste $(Z=-0.01 ; p=$ $0.89)$, nem no reteste $(Z=-0.08 ; p=0.39)$. Na análise da confiabilidade teste e reteste, verificaram-se altos valores do coeficiente de correlação intraclasse, para a média das medidas para cada um dos quatro avaliadores $(C C I \geq$ 0.98) (Tabela 2).

Tabela 1

Valores registrados do tempo (em segundos) por avaliador no teste de agilidade para o voleibol sentado $(n=8)$.

\begin{tabular}{ccccc}
\hline \multirow{2}{*}{ Avaliador } & \multicolumn{2}{c}{ Mediana (AIQ) } & \multicolumn{2}{c}{ Mediana (AIQ) } \\
\cline { 2 - 5 } & \multicolumn{2}{c}{ Teste } & Deteste \\
\cline { 2 - 5 } & Direita & Esquerda & $6.24(1.27)$ & $6.26(1.32)$ \\
A & $6.39(1.41)$ & $6.43(1.40)$ & $6.28(1.33)$ & $6.32(1.39)$ \\
B & $6.26(1.42)$ & $6.22(1.36)$ & $6.27(1.24)$ & $6.30(1.31)$ \\
C & $6.29(1.74)$ & $6.26(1.36)$ & $6.17(1.22)$ & $6.23(1.33)$ \\
D & $6.29(1.43)$ & $6.34(1.45)$ & $6.24(1.20)$ & $6.28(1.27)$ \\
Todos & $6.31(1.36)$ & $6.31(1.32)$ & &
\end{tabular}

Nota: Dados expressos por mediana e amplitude interquartil (AIQ).

Tabela 2

Confiabilidade teste e reteste (repetibilidade) do teste de agilidade para o voleibol sentado $(n=8)$.

\begin{tabular}{|c|c|c|c|c|c|}
\hline \multirow[b]{2}{*}{ Avaliador } & \multirow[b]{2}{*}{ CCI (IC95\%) } & \multicolumn{2}{|c|}{ Limites de concordância de 95\% (Bland-Altman) } & \multicolumn{2}{|c|}{ Valor- $p$ (Wilcoxon) } \\
\hline & & $\begin{array}{c}\text { Teste/reteste } \\
\text { Direita } \\
\end{array}$ & $\begin{array}{c}\text { Teste/reteste } \\
\text { Esquerda }\end{array}$ & $\begin{array}{c}\text { Teste/reteste } \\
\text { Direita } \\
\end{array}$ & $\begin{array}{c}\text { Teste/reteste } \\
\text { Esquerda }\end{array}$ \\
\hline A & $0.992(0.976-0.998)$ & -0.425 a 0.738 & -0.679 a 1.00 & 0.195 & 0.250 \\
\hline $\mathrm{B}$ & $0.993(0.978-0.998)$ & -0.676 a 0.636 & -0.811 a 0.623 & 0.844 & 0.742 \\
\hline $\mathrm{C}$ & $0.989(0.968-0.998)$ & -0.776 a 0.808 & -0.855 a 0.765 & 0.742 & 1.000 \\
\hline $\mathrm{D}$ & $0.985(0.955-0.997)$ & -0.743 a 0.985 & -0.985 a 1.21 & 0.641 & 0.297 \\
\hline
\end{tabular}

Nota: CCI= Coeficiente de correlação intraclasse. 
Em relação à confiabilidade interavaliador, não foram verificadas diferenças significativas entre avaliadores, para ambos os lados, tanto para o teste $(p>0.05)$, quanto para o reteste ( $p>$ 0.05) (Tabela 3). Ainda conforme a Tabela 3, verifica-se excelente reprodutibilidade das medidas interavaliador, tanto para o teste (CCI $\geq$ 0.99) quanto para o reteste $(C C I \geq 0.98)$, cujo erro padrão de medida foi baixo nas duas situações, independente do par de avaliador comparado.

Tabela 3

Confiabilidade interavaliador (reprodutibilidade) do teste de agilidade para o voleibol sentado $(n=8)$.

\begin{tabular}{|c|c|c|c|c|c|c|c|c|c|c|}
\hline \multirow{4}{*}{ 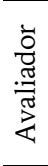 } & \multirow{2}{*}{\multicolumn{2}{|c|}{$\mathrm{CCI}\left(\mathrm{IC}_{95 \%}\right)$}} & \multicolumn{4}{|c|}{ Bland-Altman } & \multirow{2}{*}{\multicolumn{4}{|c|}{$\begin{array}{c}\text { Valor- } p \\
\text { (Kruskal-Wallis) }\end{array}$}} \\
\hline & & & & ro & Limites de conc & cordância $(95 \%)$ & & & & \\
\hline & \multirow{2}{*}{ Teste } & \multirow{2}{*}{ Reteste } & \multirow{2}{*}{ Teste } & \multirow{2}{*}{ Reteste } & \multirow{2}{*}{ Teste } & \multirow{2}{*}{ Reteste } & \multicolumn{2}{|c|}{ Teste } & \multicolumn{2}{|c|}{ Reteste } \\
\hline & & & & & & & Direita & Esquerda & Direita & Esquerda \\
\hline$\stackrel{\infty}{\star}$ & $\begin{array}{c}0.995 \\
(0.986-0.998)\end{array}$ & $\begin{array}{c}0.990 \\
(0.970-0.996)\end{array}$ & 0.168 & 0.048 & -0.230 a 0.566 & -0.397 a 0.300 & & & & \\
\hline$\underset{凶}{凶}$ & $\begin{array}{c}0.994 \\
(0.982-0.998)\end{array}$ & $\begin{array}{c}0.986 \\
(0.959-0.995)\end{array}$ & 0.135 & 0.039 & -0.175 a 0.445 & -0.318 a 0.240 & & & & \\
\hline$\stackrel{\theta}{x}$ & $\begin{array}{c}0.996 \\
(0.988-0.999)\end{array}$ & $\begin{array}{c}0.982 \\
(0.949-0.994)\end{array}$ & 0.096 & 0.052 & -0.150 a 0.343 & -0.226 a 0.331 & & & & \\
\hline$\underset{\star}{u}$ & $\begin{array}{c}0.992 \\
(0.978-0.997)\end{array}$ & $\begin{array}{c}0.990 \\
(0.971-0.997)\end{array}$ & 0.033 & 0.009 & -0.362 a 0.296 & -0.269 a 0.288 & 0.978 & 0.961 & 0.997 & 0.997 \\
\hline$\underset{x}{\infty}$ & $\begin{array}{c}0.994 \\
(0.983-0.998)\end{array}$ & $\begin{array}{c}0.987 \\
(0.963-0.995)\end{array}$ & 0.072 & 0.101 & -0.447 a 0.304 & -0.311 a 0.513 & & & & \\
\hline $\begin{array}{l}\theta \\
\dot{x} \\
u\end{array}$ & $\begin{array}{c}0.993 \\
(0.979-0.997)\end{array}$ & $\begin{array}{c}0.983 \\
(0.951-0.994)\end{array}$ & 0.038 & 0.091 & -0.302 a 0.225 & -0.163 a 0.346 & & & & \\
\hline
\end{tabular}

Nota: $\mathrm{CCI}=$ Coeficiente de correlação intraclasse.

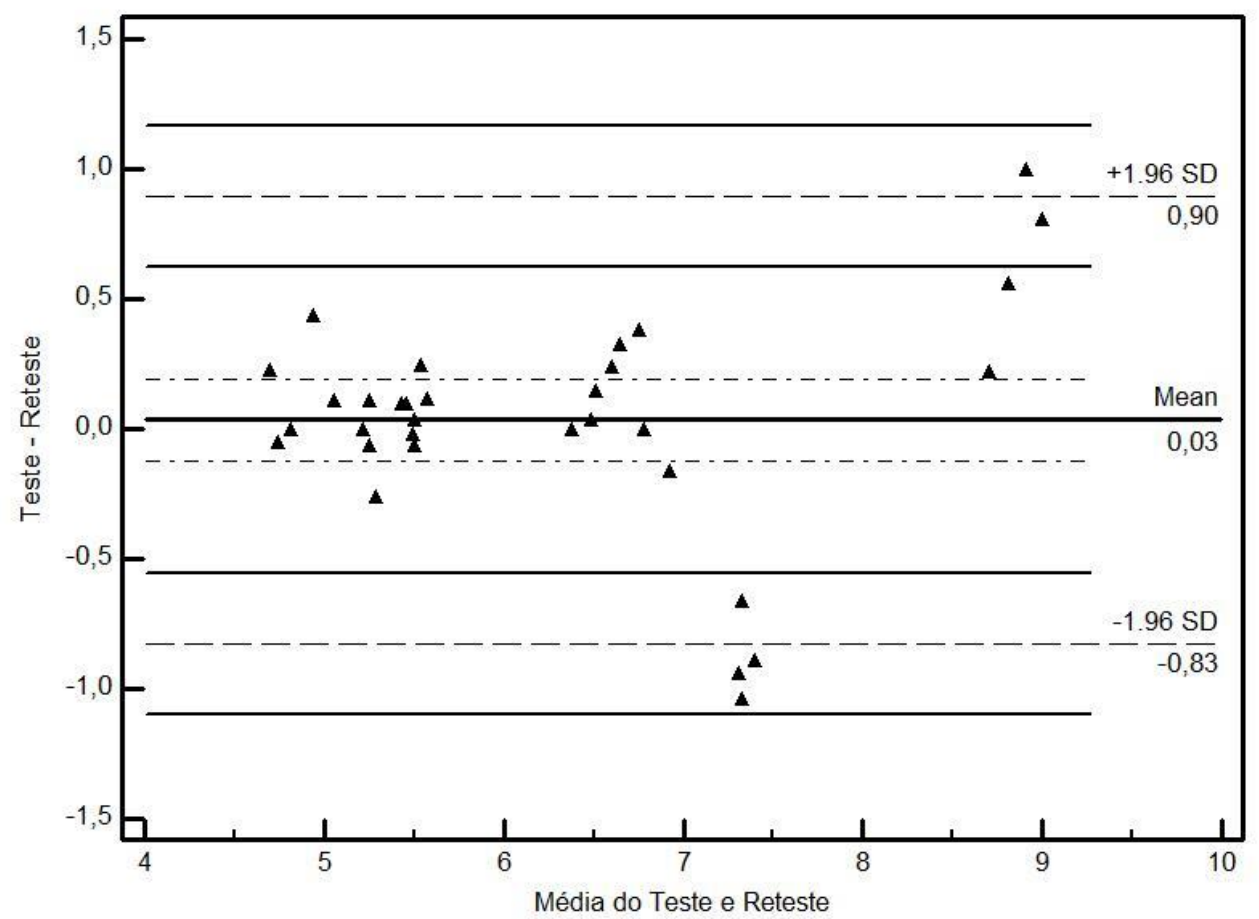

Figura 3. Gráfico de Bland-Altman para a concordância teste e reteste para todos os avaliadores, com medidas do lado direito e esquerdo agrupadas. 
Os limites de concordância apontam que as diferenças entre medidas de teste e reteste foram de 0.9 segundo, verificando-se tanto alta concordância (0.94), quanto acurácia (0.99). Salienta-se que apenas quatro medidas apresentaram-se fora dos limites inferior e superior no método de Bland-Altman (Figura 3).

Quanto ao entendimento em relação a descrição do teste (clareza), sua aplicabilidade e sobre o fato de o teste TAVS mensurar a agilidade, os cinco especialistas (100.0\%) foram unânimes em defini-lo como de entendimento muito fácil, muito viável para a modalidade, e capaz de medir a agilidade para a situação específica da modalidade. Assim, os resultados colaboram com a validação do conteúdo.

\section{DISCUSSÃO}

O desenvolvimento de testes, voltados para a medida dos componentes da aptidão física, tem surgido como uma ferramenta prática para a avaliação e controle do treinamento em diferentes populações. Entretanto, parece haver uma escassez de estudos que empregam estas medidas para as modalidades paralímpicas (Gorgatti \& Böhme, 2003; Molik et al., 2010; Wiś niowska et al., 2012; Yilla \& Sherrill, 1998). Nesse sentido, este estudo investigou a confiabilidade teste e reteste, confiabilidade interavaliador e validade de conteúdo de um Teste de Agilidade para o Voleibol Sentado (TAVS). Nossos resultados demonstraram que o TAVS aplicado por diferentes avaliadores, em ocasiões distintas, apresentou altos valores de confiabilidade, com baixo erro, indicando que o teste pode ser considerado uma alternativa viável para mensurar a agilidade no voleibol sentado. Em relação à validação de conteúdo, os especialistas definiram o teste como viável e capaz de medir a agilidade dos jogadores de voleibol sentado.

Verificou-se, também, que o lado de início da execução da tentativa (direito ou esquerdo) não influencia no escore do teste. Em adição, o fato de existirem pequenas variações sistemáticas nos valores das tentativas entre avaliadores, nas duas ocasiões, sugere que o TAVS pode ser usado com grande confiança por um único avaliador.
É importante ressaltar que a análise confiável destes testes consiste em uma necessidade, tanto para técnicos, quanto para classificadores (Medeiros et al., 2012; Tweedy \& Vanlandewijck, 2011; Vute \& Urnaut, 2009). Os limites de concordância apontam que as diferenças entre medidas de teste e reteste parecem ser menores do que 1 segundo. Algumas razões podem ter contribuído para a alta confiabilidade e acurácia teste e reteste encontrada neste estudo (McBride, 2005). Inicialmente, destaca-se que as sessões de teste e reteste para a medida da agilidade foram separadas por 48 horas. Este intervalo pode prevenir tanto os efeitos de aprendizado, quanto da fadiga dos sujeitos sobre a reprodutibilidade destas medidas. Além disso, o uso objetivo do protocolo, explicações, posicionamento e ambiente durante as duas sessões de testagem podem ter colaborado para a consistência das medidas no TAVS. Pode-se verificar, ao preceder as sessões por uma tentativa de reconhecimento do percurso, mesmo que de forma lenta, a minimização do efeito do aprendizado.

Percebe-se, contudo, que outros fatores estão associados à agilidade, sobretudo, referente ao voleibol sentado. O posicionamento das mãos, por exemplo, é essencial não somente para melhorar a força de propulsão contra o solo e, por consequência, a velocidade do movimento, mas para fazer com que os atletas tenham reações mais rápidas e adequadas para coordenar as ações na realização do contato na bola, de acordo com a situação de jogo (Singhal et al., 2013; Zerger, 2008). Em um estudo recente de Singhal et al. (2013), a posição das mãos lateral ao corpo no solo permitiu que os atletas se movimentassem de maneira mais rápida, quando comparada com as mãos em outras direções. Desta forma, é provável que atletas de elite consigam empregar melhor suas mãos para empurrar o solo, com alguma contribuição dos membros inferiores e tronco, a fim de vencer a inércia (Zerger, 2008), melhorando assim o tempo dos deslocamentos.

Além disso, a habilidade de "ler o jogo" é muito significativa para este desporto, uma vez que as decisões devem ser tomadas, muitas vezes, a partir das ações do adversário. Assim, fatores relacionados à habilidade visual e à 
destreza percetiva e cognitiva são fundamentais aos atletas desta modalidade (Piras, Lobietti, \& Squatrito, 2010; Sibley \& Etnier, 2004). Um estudo recente de Piras, Lobietti, e Squatrito (2014) demonstrou que atletas de voleibol sentado mais experientes têm maior capacidade de codificar de forma eficiente a informação específica do domínio que é relevante para a tarefa. Nesse sentido, especula-se que atletas de elite apresentem maior agilidade no TAVS, por compreenderem a tarefa com melhor qualidade.

No voleibol sentado, são poucas as ferramentas desenvolvidas para análise de uma habilidade específica para o jogo (Molik et al., 2008; Singhal et al., 2013). Na investigação de Molik et al. (2008), testes de velocidade, potência e agilidade foram aplicados, sugerindo uma bateria simples para a modalidade. Entretanto, a distância sugerida para o teste de agilidade é elevada, incompatível com as peculiaridades do voleibol sentado, uma vez que os autores utilizaram uma metragem adaptada do basquetebol. Além disso, estes autores não analisaram características psicométricas dos testes, limitando a discussão com o presente estudo.

A validade de um teste é frequentemente realizada pela comparação de medidas com testes equivalentes a um "padrão ouro". Contudo, não se tem um teste desta natureza para os atletas de voleibol sentado. Uma vez que testes de campo respeitam a especificidade das exigências da modalidade, o TAVS pode ser considerado um instrumento de avaliação relevante por promover informações consistentes sobre a atuação do atleta. Nos estudos de Gorgatti e Böhme (2003) e Pasetto et al. (2011), a validação por conteúdo dos instrumentos foi realizado por comissões julgadoras formada por especialistas. Dessa forma, a utilização desta estratégia e os resultados encontrados demonstram que os técnicos de voleibol sentado terão facilidade na aplicação do TAVS.

Em outras modalidades paralímpicas, a validação de instrumentos específicos já tem ocorrido na literatura, ainda de forma escassa, como no caso do rugby em cadeiras de rodas, através da bateria de Beck (Yilla \& Sherrill, 1998) e no basquetebol em cadeira de rodas, com os estudos de validação de um teste específico de agilidade para adultos (Gorgatti \& Böhme, 2003) e crianças (Pasetto et al., 2011), e a bateria de habilidades em diferentes domínios de de Groot, Balvers, Kouwenhoven, e Janssen (2012). Portanto, o TAVS parece ser uma alternativa aos testes já existentes para monitorar a intervenção em programas dirigidos para melhorar $\mathrm{o}$ desempenho de jogadores de voleibol sentado.

Ao mesmo tempo em que o uso de cronómetros manuais caracteriza o TAVS como um teste de campo próximo da realidade da maioria dos treinadores, trata-se de uma das limitações desta investigação. O uso de células fotoelétricas, provavelmente, pode ser uma alternativa interessante para o registro do tempo, reduzindo as pequenas diferenças intra-avaliador e interavaliador, melhorando a precisão da medida. Em adição, a fim de melhorar aspetos qualitativos do teste, sugere-se analisar o tempo não somente no percurso total, mas considerando as parciais dos deslocamentos laterais, muito relevantes para a modalidade. No entanto, o fato de existirem poucos estudos similares limita a discussão em relação aos escores do TAVS.

Considerando-se que o voleibol sentado é recente no Brasil e está em expansão de forma difusa em vários Estados, pesquisas com esta população têm limitações decorrentes da dificuldade de contato com a amostra e da maturação desportiva das equipas, as quais podem ser consideradas, em grande parte, como iniciantes. Embora a classificação funcional não seja o único fator que determina proficiência, o fato de haver atletas em condições motoras diferentes em nossa amostra, impossibilitou a criação de um modelo padrão de referência, uma vez que a perda funcional causada pela deficiência motora não foi controlada. Entretanto, na seleção amostral, a experiência dos voluntários foi um critério uniforme.

Apesar disso, os dados aqui levantados oferecem informações relevantes que podem servir de subsídio para treinadores e comissão técnica na elaboração de suas atividades, de modo a controlar mais variáveis do treinamento 
e buscar desempenhos competitivos mais elevados. Destaca-se que o TAVS é um protocolo de campo, de baixo custo e de fácil aplicabilidade nos contextos indoor, uma vez que possibilita ao técnico, sem o uso de alta tecnologia, a sua execução. Da mesma forma, devido à sua especificidade, o TAVS apresenta grande relação com as habilidades técnicas desempenhadas no voleibol sentado, mas, seria pertinente uma avaliação dos jogadores por posição. Desta forma, especula-se, também, a possibilidade do uso conjunto do TAVS com habilidades técnicas relacionadas ao voleibolista com deficiência motora.

\section{CONCLUSÃO}

Os achados do presente estudo sugerem que o TAVS é um teste confiável, com adequada validade de conteúdo, indicando uma proposta viável para mensurar a agilidade no voleibol sentado. Devido à sua simplicidade e baixo custo, a aplicação do TAVS pode ser uma alternativa aos testes já existentes para monitorar a intervenção em programas dirigidos para melhorar $\mathrm{o}$ desempenho de jogadores de voleibol sentado. Considerando-se o número limitado de sujeitos na amostra e, também, que aspetos cognitivos e percetivos contribuem para o desempenho da agilidade, estudos futuros devem ser conduzidos para confirmar os atuais resultados. Sugere-se, em análises futuras, que os jogadores possam ser comparados por especialidade de posição.

\section{Agradecimentos:}

Ministério do Esporte/Brasil

\section{Conflito de Interesses:}

Os autores Elaine Cappellazzo Souto e Leonardo dos Santos Oliveira contribuíram igualmente para este manuscrito.

\section{Financiamento:}

Nada a declarar

\section{REFERÊNCIAS}

Brasile, F. M. (1986). Wheelchair basketball skills proficiencies versus disability classification. Adapted Physical Activity Quarterly, 3(1), 6-13.

Brasile, F. M. (1990). Performance evaluation of wheelchair athletes: More than a disability classification level issue. Adapted Physical Activity Quarterly, 7(4), 289-297.

Correction: A Note on the Concordance Correlation Coefficient. (2000). Correction: A Note on the Concordance Correlation Coefficient. Biometrics, 56(1), 324-325.

de Groot, S., Balvers, I. J. M., Kouwenhoven, S. M., \& Janssen, T. W. J. (2012). Validity and reliability of tests determining performance-related components of wheelchair basketball. Journal of Sports Sciences, 30(9), 879-887. http://doi.org/10.1080/02640414.2012.675082

Gorgatti, M. G., \& Böhme, M. T. S. (2003). Autenticidade científica de um teste de agilidade para indivíduos em cadeira de rodas. Revista Paulista de Educação Física, 17(1), 41-50.

Hasanbegović, S., Ahmetović, S., \& Dautbasic, S. (2011). Effects of programmed training on motor abilities of persons with movement impairment in sitting volleyball. Homo Sporticus, 13(1), 6871.

McBride, G. B. (2005). A proposal for strength-ofagreement criteria for Lin's Concordance Correlation Coefficient (NIWA Client Report, HAM2005-062.). Hamilton, New Zealand: National Institute of Water \& Atmospheric Research Ltd.

Medeiros, A., Ribeiro, A., \& de Oliveira, R. G. (2012). Voleibol sentado. Em M. T. Mello \& C. Winckler (Eds.), Esporte paralímpico (1st ed., pp. 220231). São Paulo: Atheneu Editora.

Molik, B., Kosmol, A., \& Skucas, K. (2008). Sport specific and general sporting physical fitness of sitting volleyball athletes. Physiotherapy, 16(4), 68-75. http://doi.org/10.2478/v10109-0090047-2

Morrow, J. R., Jackson, A. W., Disch, J. G., \& Mood, D. P. (2014). Medida e Avaliação do Desempenho Humano (4th ed.). Porto Alegre: Artmed Editora.

Nelson, J. K., Thomas, J. R., \& Silverman, S. J. (2012). Métodos de pesquisa em atividade física (6th ed.). Porto Alegre: ARTMED.

Pasetto, C. V. F., Barros, T., Mello, M. T., Orbetelli, R., Greguol, M., Interdonato, G. C., \& Greguol, M. (2011). Validação do teste de agilidade em ziguezague para crianças com deficiência física - doi: 10.4025/reveducfis.v22i2.9334. Journal of Physical Education, 22(2), 169-176. http://doi.org/10.4025/reveducfisv22n2p169176

Piras, A., Lobietti, R., \& Squatrito, S. (2010). A study of saccadic eye movement dynamics in volleyball: comparison between athletes and non-athletes. The Journal of Sports Medicine and Physical Fitness, 50(1), 99-108. 
Piras, A., Lobietti, R., \& Squatrito, S. (2014). Response time, visual search strategy, and anticipatory skills in volleyball players. Journal of Ophthalmology, 2014, 189268. http://doi.org/10.1155/2014/189268

Protić, M., \& Valkova, H. (2011). Psychosocial aspects of player's engagement to the sitting volleyball. Acta Kinesiologica, 5, 12-16.

Semenick, D. (1990). The T-test. National Strength \& Conditioning Association Journal, 12(1), 36-37.

Sheppard, J. M., \& Young, W. B. (2006). Agility literature review: Classifications, training and testing. Journal of Sports Sciences, 24(9), 919932.

http://doi.org/10.1080/02640410500457109

Sibley, B. A., \& Etnier, J. L. (2004). Time Course of Attention and Decision Making during a Volleyball Set. Research Quarterly for Exercise and Sport, 75(1), 102-106. http://doi.org/10.1080/02701367.2004.106091 38

Singhal, K. L. S., Hwang, G. Y., Davis, R., \& Kwon, Y.H. (2013). Effects of Two Starting Floor Hand Positions on Movement Patterns of Elite Sitting Volleyball Players. Palaestra, 27(2).
Soše, J. (2009). Canonical relations between cognitive abilities and specific motor abilities of sittingvolleyball players. Homo Sporticus, 11(2), 6-9.

Tweedy, S. M., \& Vanlandewijck, Y. C. (2011). International Paralympic Committee position stand-background and scientific principles of classification in Paralympic sport. British Journal of Sports Medicine, 45(4), 259-269. http://doi.org/10.1136/bjsm.2009.065060

Vute, R. (1999). Scoring skills performances of the top international men's sitting volleyball teams. Gymnica, 29(2), 55-62.

Vute, R., \& Urnaut, A. G. (2009). Teaching and coaching volleyball for the disabled: foundation course handbook (2nd ed.). Ljubljana: Faculty of Education.

Wiśniowska, M., Tasiemski, T., \& Bauerfeind, J. (2012). Athletic Identity Assessment in Disabled Sitting Volleyball Players. Physiotherapy/Fizjoterapia, 20(1), 10-19.

Yilla, A., \& Sherrill, C. (1998). Validating the Beck battery of quad rugby skill tests. Adapted Physical Activity Quarterly, 2(15), 155-167.

Zerger, M. (2008). A study of movement in sittingvolleyball (Master in Kinesiology and Health Studies). University of Central Oklahoma, Okhlahoma.

Todo o conteúdo da revista Motricidade está licenciado sob a Creative Commons, exceto quando especificado em contrário e nos conteúdos retirados de outras fontes bibliográficas. 